\title{
Use \& Misuse of Water-filtered Tobacco Smoking Pipes in the World. Consequences for Public Health, Research \& Research Ethics
}

\author{
Kamal Chaouachi*
}

DIU Tabacologie, Université Paris XI, France

\begin{abstract}
Background: The traditional definition of an "epidemic" has been revisited by antismoking researchers. After 400 years, Doctors would have realized that one aspect of an ancient cultural daily practice of Asian and African societies was in fact a "global "epidemic"". This needed further investigation particularly if one keeps in his mind the health aspects surrounding barbecues. Method: Here, up-to-date biomedical results are dialectically confronted with anthropological findings, hence in real life, in order to highlight the extent of the global confusion: from the new definition of an "epidemic" and "prevalence" to the myth of "nicotine "addiction"” and other themes in relation to water filtered tobacco smoking pipes (WFTSPs). Results: We found that over the last decade, many publications, -particularly reviews, "metaanalyses" and "systematic reviews"- on (WFTSPs), have actually contributed to fuelling the greatest mix-up ever witnessed in biomedical research. One main reason for such a situation has been the absolute lack of critical analysis of the available literature and the uncritical use of citations (one seriously flawed review has been cited up to 200 times). Another main reason has been to take as granted a biased smoking robot designed at the US American of Beirut whose measured yields of toxic chemicals may differ dozens of times from others' based on the same "protocol". We also found that, for more than one decade, two other main methodological problems are: 1) the long-lived unwillingness to distinguish between use and misuse; 2) the consistent unethical rejection of biomedical negative results which, interestingly, are quantitatively and qualitatively much more instructive than the positive ones. Conclusion: the great majority of WFTSP toxicity studies have actually measured, voluntarily or not, their misuse aspects, not the use in itself. This is in contradiction with both the harm reduction and public health doctrines. The publication of negative results should be encouraged instead of being stifled.
\end{abstract}

Keywords: Electronic cigarette, harm reduction, hookah, narghile, public health,shisha, smoking, tobacco.

\section{INTRODUCTION}

This is likely the first time in the annals of medicine that a several century-old cultural invention, being simultaneously a peculiar trait of many African and Asian societies, is described in the biomedical literature of the last decade as an "epidemic" and even a global one [1]. From an epistemological standpoint, it is worth stressing that in a not so remote past, both anthropological and biomedical research findings about the domesticated health aspects of the practice used to agree with each other. However, a sudden change has occurred in 2002. The main reason is the sudden adoption, from that year onwards, of a reductionist approach whereby the highly complex socio-cultural matrix of a human daily practice, namely the use and misuse of water filtered tobacco smoking pipes (WFTSPs) across the world, has virtually been overlooked. Interestingly, a recent scholarly article denounces for the first time the existence of "biomedical reductionism in tobacco control" (sic) [2].

On one hand, a so-called laboratory "model" of the corresponding human practice was designed at the US-AUB (US American University of Beirut). It is actually a mere smoking robot whose relevance has been widely, although blindly, accepted by many as "standardised" [1]. On the other hand,

*Address correspondence to this author at the DIU Tabacologie, Université Paris XI, 63 avenue Gabriel Peri, 94276 Le Kremlin-Bicêtre, France; Tel: 3314959 6617; Fax: 3315839 3695; E-mail: kamcha@gmail.com all WFTSPs, in spite of their well known material and sociocultural diversity, have been arbitrarily lumped together under a same umbrella. A neologism was invented for this purpose: ««waterpipe»» in one word. From the outset, we have stressed that such a methodological move amounted to a form of unscientific reductionism whose direct outcome is a global confusion regarding concepts and objects alike. What has also gone unnoticed so far is that a clear line has never been drawn between the actual use of these pipes and their misuse. In many instances, such a line reflects the classical distinction between traditional $v s$. modern use. If one compares with the usual approach when it comes to other aspects of everyday's life -from the (mis) use of knives to the eating of grilled vegetables or meat in traditional (or modern) devices like barbecues- one may be tempted to conclude to double standards. The latter example is even more relevant that it also entails the generation of smoke and the corresponding misuse should also raise health concerns regarding its potential toxicity $[3,4]$. Unfortunately, it seems here that when things relate to tobacco use and abuse, human passions or economic interests take over the scientific method.

Overlooking the striking diversity of WFTSPs represents a serious ethical and methodological error since all water pipes (in two words) of the world have almost nothing in common but that ««waterpipe»»" name arbitrarily imposed one decade ago [5]. From there, ««waterpipe»»)-labelled clinical studies, or those carried out in a "real" "natural" environment, have generally mixed up different products used 
by the smokers: e.g., plain tobacco of the tumbak, ajamy or tutun type with the popular flavoured moassel (tabamel), etc (See Figs. 1, 2, 3). Yet, the smoke chemistry of both (or more) types is completely different in each case and results in different health effects. What did also exacerbate the global confusion, is that the corresponding researchers, in an endeavour to demonstrate that the claimed health effects (e.g., on lung function) are supported by other studies, often blindly refer to the above mentioned US-AUB' smoking robot. Yet, they do not realise, or simply wish to ignore, how biased are the corresponding procedures which led to the imposition of the puffing machine in question $[6,7]$. Indeed, it was recently stressed how two smoking machines, in two different laboratories from two different countries (Germany and Lebanon) could produce acrolein and benzo[a]pyrene levels 66 and 20 times different, respectively, in each case [5]. Yet, the two robots were set with similar (biased) parameters: notably an exaggerated inter-puff time interval of $17 \mathrm{~s} / 20 \mathrm{~s}$; the charcoal (non-natural) literally burning the flavoured smoking mixture instead of just heating it (because of its arbitrarily fixed position atop the bowl, for one full hour). More recently, such a hazardous chemical as phenol was quantified at levels 18 times lower than those previously produced by the US-AUB's robot $(3.21 \mu \mathrm{g} v s .58 .03 \mu \mathrm{g})$ [8]. Interestingly, a common cigarette, used as a reference in similar experiments, generates (in only a few minutes) two times the phenol level produced by a shisha (over one full hour)... If one also bears in mind that while a common cigarette smoker may consume 20 units a day, and that shisha is generally smoked, according to recent epidemiological data, 1 to 3 times per week (i.e. a frequency of 0.14 to 0.43 pipe per day), then the abuse of toxicity comparisons between cigarettes and WFTSPs is blindingly obvious.

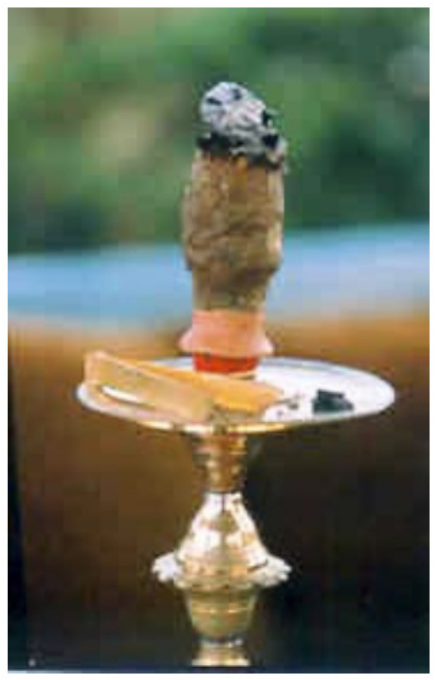

Fig. (1). Tumbak (ajamy), a classical" product in Asia and Africa. It is close to the one used by the volunteers in Mohammad et al.' study on their lung function [18]. Main characteristic: direct contact between the charcoal and the tobacco plant.

The question that arises is: how have researchers been able to trust so easily similar instruments (robots) and then feel entitled to warn against alarming levels of hazardous chemicals supposedly present in "««waterpipe»»" smoke"? The answer is that there is a clear agenda behind such an "activism"(sic) [9]. The so-called scientific "evidence" is necessary for ongoing legislation in dire straits because WFTSP now represents, according to antismoking researchers themselves, a major obstacle towards a "tobacco-free word". For them, a "tobacco "control" policy" undoubtedly represents "an effective step towards eradication"(sic) $[9,10]$.

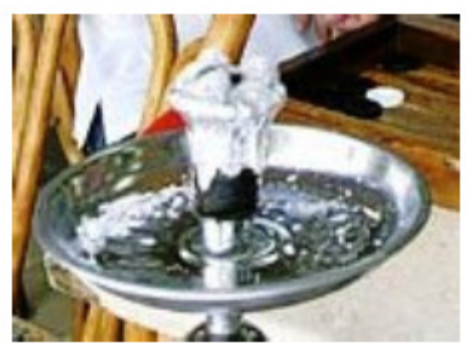

Fig. (2). Moassel (tabamel), a mixed product between tumbak and jurak (see reference book)[1]. The chemistry of its smoke is completely different from that of tumbak whose study has been prevented by the use of the unscientific confusion-fuelling neologism ««waterpipe»». Main characteristic: no direct contact between the charcoal and the smoking mixture.

Not only has the definition of an "epidemic" been revisited, as pointed out before, but also that of prevalence and psycho-pharmacological addiction as we will see further down. The answer to how such a global confusion, never witnessed before and elsewhere in the biomedical field [5], has been possible, certainly deserves a tentative answer; perhaps of a sociological nature [2]. In fact and with the benefit of hindsight, we can now state that a great number of researchers working on the hot issue of WFTSPs have got a poor grasp of the basics of tobacco science, not to mention of the everyday's life material culture of Asian and African societies. To make things worse, they often lack both skills in the same time even when some of them are physicians or biochemists native of those regions [1]. Yet, cumulated negative results (i.e. findings showing that WFTSPs are not associated with all the new alarmist risks) published in peerreviewed journals over the past decades, and more recent ones, are numerous and exceeding, in quality at least, the purported positive results, in spite of a now permanent stifling of debate [11-18]. "«Waterpipe»» antismoking teams always find a way round to avoid mentioning negative results (e.g. during the "selection" of bibliographical references when preparing "reviews", "systematic" or not, and other "meta-analyses". They may also be embarrassed when antismoking researchers themselves come up with such negative results as this happened with a toxicity study from the United Kingdom involving a smoking robot similar to the US-AUB one. Given that the results openly collide totally with those widely advertised by the US-AUB, the study has simply sunk into oblivion. Their authors, apparently embarrassed, downplayed the importance of their own findings [19].

Interestingly, in the recent debate over electronic cigarettes, "tobacco harm reduction" advocates, supposed to benefit from a large experience of exposing the prevalent shoddy science of "tobacco "control"" research, have echoed in their turn, and without the least critique, the claims against flavoured shisha. Amazingly, they did not realise that the 
smoke of the latter (when prepared correctly, not according to the US-AUB "method") is much more similar to the vapour of E-cigarettes than the smoke of regular ones [20].

\section{USE AND MISUSE OF WATER FILTERED TO- BACCO SMOKING PIPES (WFTSPS)}

It has been already made clear that the biochemical and anthropological aspects of both water filtered and cigarette smoking (particularly regarding the so-called second-hand smoke) are completely different from each other [20, 21]. For many years and in tune with a global prohibitionist agenda -as implicitly reflected in the clauses of the FCTC ("Framework Convention for "Tobacco Control") [10, 22], the mainstream media have, in an uncritical way, echoed "«"waterpipe»») experts' claims that the health effects of a common shisha smoking session are 100, 200 (and even more...) worst than those caused by regular cigarettes [23]. Further to relevant necessary critiques $[5,11,20,24]$, the new motto has suddenly become that both smokes "have ["only"] similar negative effects"... For the follower of such a mind-boggling "public health" issue, not only has the toxicity ratio suddenly been divided by a factor of at least $100 \ldots$, but such a new statement remains as false and unscientific as the previous one.... Instead of a public apology, ««waterpipe»») antismoking researchers themselves are now advising to each other not to compare anymore hookahs with cigarettes in such an arithmetic way [25]. However, the new "equivalence" (1:1 instead of 1:100) still represents a gross exaggeration because the chemistry of shisha smoke is so different and its matrix so much less complex than the one generated by a regular cigarette: dozens of times less concentrated, sometimes by two orders of magnitude. Indeed, only some 150 chemicals have been officially identified so far $v s$. 5,000 for cigarette [20]. Further to our one-decade long relentless emphasis on this point [20], a few antismoking experts now begin to openly admit the fact and echo it in their turn $[8,26]$. For example, nitrosamines are likely the most hazardous chemicals present in tar. Interestingly, and when compared with cigarette smoke, these products are consistently found (when they actually reach detection levels) in much lower concentrations in human hookah smokers, even when the water inside the tank has not been changed as is often the case (voluntarily or not) in a long series of biased studies carried out by antismoking teams [5, 27-29]. Most of the time, what has been actually measured in experimental toxicity or clinical studies, is either the result of: blatant biases (e.g. smoking machines cited as "realistically" mimicking the complex use of WFTSPs [5-7]); heavy use as in some Egyptian studies (in which some patients may regularly sit for 5 pipes and more a day, a smoking pattern barely observed outside Asia and Africa [1]); misuse (by inexperienced users still unfamiliar with a four-century old technique [1]); and/or poor hygiene [5]. Perhaps would it be more correct, for the latter cases, to say "abuse" instead of "misuse", one relevant comparison being the consumption of alcohol.

If water (in the tank) had "no filtering effect", according to antismoking public claims, why has been the "detail" of changing it after each smoking session "overlooked" in so many studies? To top it all, the subjects of a recent study in California (USA) were even compelled to smoke a minimum of two pipes in the morning [27]... Furthermore, in spite of the poorly convincing results (the toxicity of hookah smoking proved to be far less important in terms of quantified metabolites of known carcinogens), the authors generally do not refrain from hyping their findings in the mainstream media [27]. Confusion in this field has assuredly reached unprecedented, unexpected degrees. A team from the US-AUB, supposed to lead ««waterpipe»») research on a global level, even believed tar can be found in the tobacco plant itself $[7,30]$, whereas the basis of tobacco science (tobaccology) makes it quite clear to first year medical students that such a chemical compound only appears once the cigarette has been lighted, never before. In these conditions, it is apparently easy to lead astray the general public and lawmakers when official research is carried out and published in the biomedical press in this way including reports published by the WHO [5, 9-10, 20, 22-24]. Researchers on drug use know well how a word like "eradication" (used in a recent article [10]) may be interpreted and tapped. When the targeted public does not distinguish between shisha tar and cigarette tar (the former being much less toxic for being produced at temperatures hundreds of degrees Celsius below those at the tip of a cigarette), it is clear that it can be easily deceived. From there, nonsense (like: "the inhaled smoke from a one-hour waterpipe session contains as much tar as up to 600 cigarettes" [31]) stated by one of the authors of the WHO flawed report [24], can be published with no reaction.

WFTSP misuse often results from the lack, or poorness, of ventilation $[1,20]$. All cases of shisha-induced Carbon Monoxide poisoning recently reported in the literature are a direct result of the lack of public health warnings (against smoking in such places as ill-ventilated bedrooms and the like), rather than because the pipes themselves would consistently or inherently cause CO intoxication. The comparison with barbecues should be kept in mind here. Less and less people get CO-poisoned when using barbecues simply because appropriate public health warnings about their use are widespread. Another relevant comparative example here is the electronic cigarette because public health officials have recently reported an elevated numbers of telephone calls to poison centres, likely due to the misuse of these new products [32]. One ethical question here is: why do shisha users (at least outside Africa and Asia where the practice is well domesticated) are not entitled to benefit from similar warnings? Furthermore, one often silenced aspect of WFTSP use is that such pipes are smoked (at least under their traditional form) much like cigars. Now, the latter are well known for producing large quantities of $\mathrm{CO}$. Research early established that cigar inhalers had markedly elevated concentrations of carboxyhaemoglobin $(13.8 \%$ and $11.8 \%$ in primary and secondary inhalers, respectively) [33]. Cigar users know that ventilation is important [20-34]. Interestingly, a relevant study in Jordan involving a wide sample (14,310 subjects), showed that the increase of arterial blood pressure and heart rate among exclusive shisha smokers remained quite modest ( 92.57 to 92.62 and 76.40 to 76.81 , respectively [14].

The same goes for most hazardous chemicals (heavy metals, etc.) which are sometimes found in users' body fluids. Given that the levels of these products in the very moassel/tabamel (the gooey smoking mixture in shisha) are much less elevated than in the tobacco rod of cigarettes, it appears that their presence in body fluids, when it occurs, is likely 


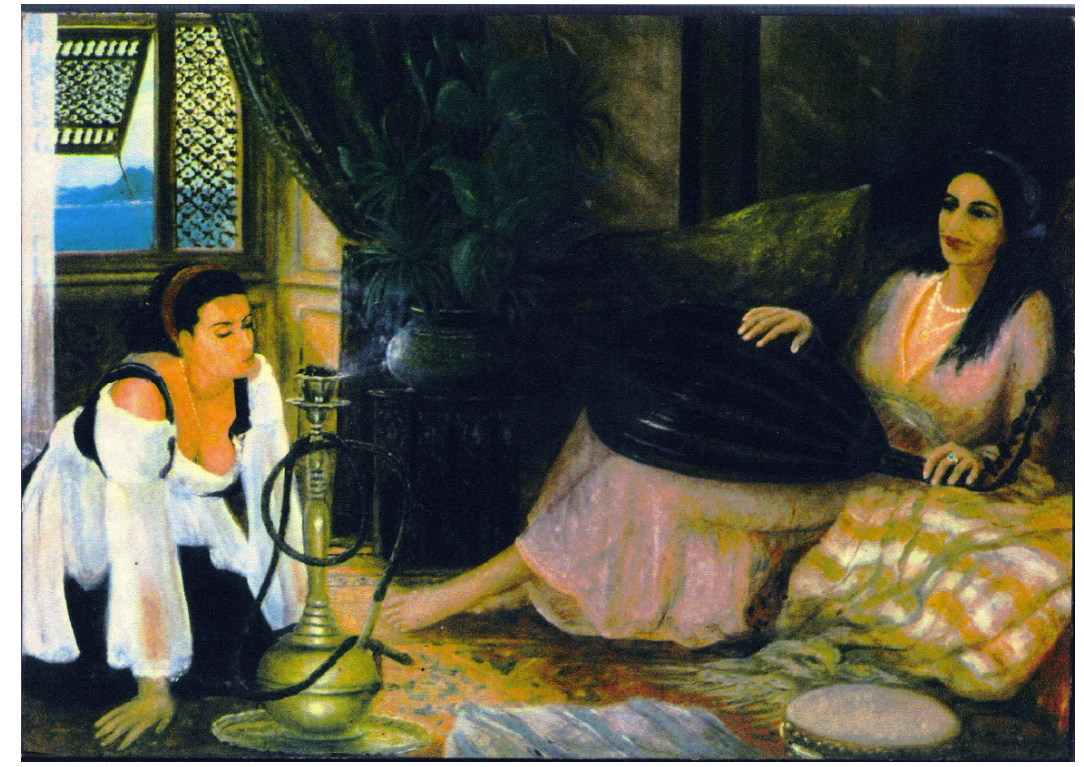

Fig. (3). Jurak, also a "classical" product in Asia and Africa. It is close to the one used by the volunteers in Sajid et al.'s study [11]. Painting depicting a Tunisian scene.

due to: the misuse of the thermal screen (aluminium, commonly of cookware quality) separating the heating source from the moassel; the metal coating of the pipes (particularly the bowl) and/or; the charcoal. As with ventilation, any public health campaign worthy of this name should have actually warned users against this problem, for more than one decade now $[1,16,20]$. Such harm reduction based recommendations also apply to benzene produced by the charcoal as in barbecues [3-4, 8, 35]. Instead of exclusively hyping the risks of inhaling benzene, WFTSP users could simply be invited as follows: "if you wish to smoke hookah, please avoid ill-ventilated places" [1, 20]. If, as the authors of an alarmist study state "there is no safe level of exposure to benzene" and even suggest to "revent" (does this also mean "ban"?) hookah smoking [35], then should not automobiles and barbecues, known to emit much larger amounts of that chemical, be also targeted by the same warnings? Olsson \& Petersson found that above charcoal for grilling, benzene concentration exceeded $10 \mathrm{mg} \mathrm{m}-3$ at a $5 \%$ carbon dioxide level [3]. In sum, what most WFTSP toxicity studies generally have shown so far to potential users is, at best, "how not to smoke" rather than the poisons they actually inhale [5].

\section{LAB TOXICITY TESTS CONSISTENTLY CON- TRADICT ACTUAL HEALTH EFFECTS ON HU- MANS}

As previously stated in the literature, the so-called great health hazards taken as granted by some researchers have been deducted from a series of biased experimental studies chiefly based on smoking machines [5-7, 20, 24]. In these conditions, it is not surprising to see that recent in-vitro studies, and others carried out in a "real"-environment or on real human smokers (not robots), are generally contradicted by independent studies led on humans [5, 14-15, 27, 35]. For instance, independent researchers in Tunisia recently found that the lung function of exclusive heavy (users of a strong jurak-like product) (See Fig. 3) was much less affected than that of chronic exclusive cigarette smokers. Although sur- rounded with some unclear areas -whereby subjects had been likely exposed to both types of smoke-, a Syrian independent group also came to not dissimilar conclusions [1718]. Amazingly, such studies were not cited by Lebanese researchers who would select only "positive" although biased already criticised results [38-40].

We have highlighted before how the yields of toxic chemicals (acrolein, phenol, benzo[a]pyrene, etc.) produced by different teams using a similar smoking robot were totally colliding between each other. For instance, Apsley et al. in the UK reported mainstream smoke CO levels ranging from 800 to $1000 \mathrm{ppm}$ "suggesting a typical CO yield of $30 \mathrm{mg}$ per smoking session. They emphasised that they were unable to detect (L.O.D. $<0.02 \mu \mathrm{g}$ ) many of the PAHs (polycyclic aromatic hydrocarbons). The same for most metals [19]. By contrast, and just to take Cobalt as an example, the German team, in its turn, suggests levels $400 \%$ greater than those measured on the US-AUB robot [8].

Other researchers have analysed heavy metals present in the moassel itself. For instance, Saadawi et al. stresses that "the average mass of the more toxic elements (As, Cd, Cr and $\mathrm{Pb}$ ) present in a hookah smoking portion of about $15 \mathrm{~g}$, is smaller than that contained in a normal cigarette" [36]. This is in agreement with another detailed analysis of trace elements led by Khater et al. and previous ones in the Middle East [16, 37] (See Fig. 6).

For benzene, in the worst case and supposing the levels had some reality (absurd hypothesis), the German antismoking team recently found $271 \mu \mathrm{g}$ [8]. This represents, without taking any methodological precaution, the "equivalent" of the volatile compound produced by six $2 \mathrm{R} 4 \mathrm{REF}$ cigarettes $(43.4 \mu \mathrm{g})$; bearing in mind, however, the earlier stressed shisha average frequency of 0.14 to 0.43 pipe per day.

The trend to highlight "positive results" and overlook negative results is patent $[5,24]$. Before year 2002 (inception of ««waterpipe»») antismoking research), almost all research 
was of the latter type. Interestingly, it has been conducted by the pioneers and greatest names of independent (from both the pharmaceutical and tobacco industries) tobacco science like Ernst Wynder, Dietrich Hoffmann, etc. [11].

Independently obtained data generally, and consistently, contradict the widely advertised laboratory toxicity levels produced by the US-AUB smoking robot. When they do not, this is generally due to confusion factors or blatant flaws as not changing the water [5, 27-29].

To top it all, the levels of toxic chemicals, most of the time, and for most of them, remain much lower than those related to cigarette use $[8,19,28,29] \ldots$ Because of the prevalent prohibition ("eradication" (sic)) paradigm [10], and the related world FCTC agenda [10, 22], many researchers who come up with negative results feel somewhat embarrassed and systematically tend to downplay them (a psychosociological process also known as cognitive dissonance). A few years back, at an international anti-tobacco conference, a study (not yet published by the time of the event) on a series of toxic carcinogenic chemicals was announced. Unsurprisingly, the levels were not higher than among cigarette smokers and, most of the time, not very different from controls. Even the level of NNAL (4-(methylnitrosamino)-1-(3pyridyl)-1-butanol), metabolite of the potent carcinogen NNK (4-(methylnitrosamino)-1-(3-pyridyl)-1-butanone), was not different from that of controls. However, the researcher who presented these negative results to the public surprisingly stated that they were "disappointing from a scientific point of view" (sic) and, for this reason, refrained from detailing all of them $[41,42]$.

If (absurd hypothesis) the toxicity tests run by the USAUB thanks to the smoking robot had some reality, then epidemiologic studies should provide high figures for risks of cancer, to start with. On the contrary, the first cancer aetiological studies on hookah smoking and cancer (which also compared risk with cigarettes) show that, despite the consumption of huge amounts of coarse tobacco of poor quality over decades, exclusive users of hookah had biological markers of cancer (CEA) much less elevated than cigarette consumers [11]. According to Indian researchers, local hookahs can be filled up to $375 \mathrm{~g}$ tobacco a day [43]. By the time the study was led, the authors did not even discuss the question of whether or not, the users (the heavy ones in particular) did change the water or not. It appeared later that, in the same region (Kashmir/Punjab), water is barely changed, not to mention its possible contamination [44]. In the light of this new information, the results of such pioneering studies speak by themselves until similar detailed solid investigations are led [11]. Amazingly, after seven years from this pioneering work, no other similar study has been published on this issue.

\section{SHISHA, E-CIGARETTES AND PASSIVE SMOK- ING}

Electronic cigarettes were first viewed as efficient harm reduction tools although later described in many antismoking publications as entailing an "unknown" "toxicity"... Interestingly, in the light of the already mentioned global confusion, few scientists working on this new product have noted that the designers of the E-cigarette actually drew their inspira- tion from the very principle of WFTSPs [20]. Furthermore, if shisha smoking had to be compared with other tobacco use methods, E-cigarettes, not regular ones, should have been the "natural" candidates from the beginning. Indeed, when correctly prepared and set up (use vs. misuse), a modern shisha does generate an aerosol more chemically similar to the (nicotine and flavours) vapour generated by an E-cigarette. Unfortunately, a great part of the global mix-up (among researchers as well as many users) is due to the fact that, unlike sealed cigarettes, it is always easy to tinkle (and users, particularly the inexperienced ones, do) with the charcoal (its nature, pressure, position above the bowl, etc.) and its contact (particularly the duration of this contact) with the smoking mixture. In each given situation, this invariably results in totally different chemical profiles for the generated aerosol. In such a biased configuration, the flavoured smoking mixture (moassel: to mention the one exclusively targeted by «"waterpipe»») antismokers) packed inside the bowl (below the aluminium thermal screen) will not - particularly in the vicinity of the geometric spot beneath the glowing embers-, be heated but literally charred and burnt with the expected negative chemical consequences (what actually happens in the US-AUB smoking robot "protocol").

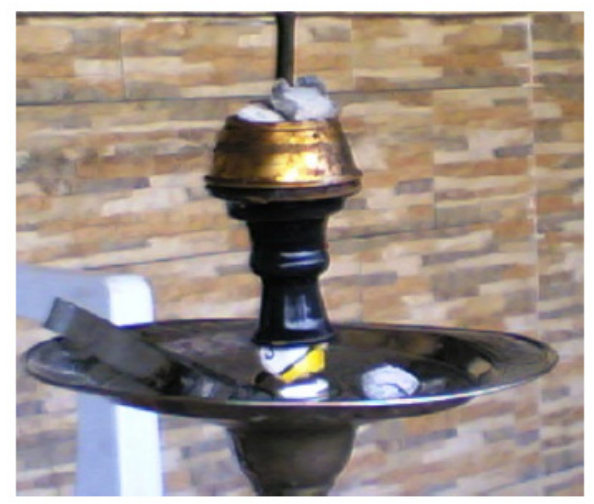

Fig. (4). Harm reduction in practice. The Tunisian inverted Keskes (sieve) is a vaporising ball in use in many cafes of the country. Its proper use needs some "training" in order to avoid causing the inverse effect (burning the moassel instead of just vaporising its active principles).

In the absence of any scientific debate, transparency and help from public health officials, it is interesting to note that WFTSP users have now successfully designed, by themselves, bowls in which the heating source (charcoal embers) is kept at bay from the flavoured smoking mixture (tabamel/moassel) thus making the inhaled aerosol even more comparable with the vapour produced by E-cigarettes (See Fig. 2, 4). Such a harm-reduction concern stems from the same basis as the one which led to the design of a noncharcoal powered shisha pipe $[6,45]$.

Unfortunately, many so-called "tobacco harm reduction" advocates ignore these facts and, while they were known in the past for being critical and even radical regarding the bad science (on regular cigarettes and smokeless tobacco) published by antismoking researchers, many of them have surprisingly endorsed the latter's biased discourse on the WFTSP "global epidemic". The case of a French team, already criticised elsewhere [20], is interesting because it re- 
cently compared the E-cigarette aerosol with that generated by a regular cigarette and shisha, however deceivingly glossing over a key chemical difference between both [46]. Indeed, it is quite deceptive to quantitatively compare cigarette and WFTPs regarding PM10, PM2.5 or nanoparticles [47]. Certainly, environmental tobacco smoke (ETS) is made up of millions of particles of different sizes but their composition (nicotine, hydrocarbons, phenols, heavy metals and glycerol) is amazingly never advertised. The chemical profile of cigarette ETS is in fact completely different from that generated by a WFTSP [20]. Consequently, such comparisons are unscientific and are obviously made to deceive the broad public and lawmakers [20]. A few years back, the case of cigarette third-hand smoke was already scientific nonsense. Here, one can figure out what such a hyping of risks amounts to when it comes to WFTSP smoke, known to be dozens of times less concentrated $[35,48]$.

\section{LACK OF EVIDENCE FOR SHISHA "NICOTINE "ADDICTION""}

The use of quotation marks is very important here because it is possible that among heavy smokers (several sessions a day) of certain types of pipes (and only certain pipes) filled with certain varieties of tobacco (not the flavoured one), some form of dependence may have remained. Of interest are also those who have switched, in a recent or more remote past, from cigarette smoking to tumbak or jurak use (See Figs. 1, 3). Unfortunately for such individuals, and for some unclear reason never described or studied, some features of their previous smoking behaviour (cigarette, tumbak, jurak) may have outlasted. However, when it comes to the now world popular flavoured smoking mixture (moassel/tabamel) (See Fig. 2), five arguments at least shatter the new dogma ("addiction"):

a There is no evidence of the existence of such a thing as shisha "nicotine "addiction"" and the best objection is that in almost all related surveys [49], the great majority ( $80 \%$ and more) of users consistently state that they can "quit" at any moment, spontaneously pointing out that they can remain several days without feeling such things as "craving" for their "drug". Here, the corresponding percentages for cigarette users should be borne in mind. They are actually the exact opposite: only some $10 \%$ are recreational while the remaining ones are addicted. Furthermore, in some of these surveys, the directed questions are asked in such a biased way (for instance: "Do you know that hookah is associated with cancer, etc. [...] and that experts have demonstrated that it is addictive too", preferably repeated several times before and during the course of the interview...) that the respondents have generally no other choice than to "admit" that they it is "true" and that they did not "realise" at first that they were actually "hooked"...

b Any observer should not downplay the effect of widespread continuous scaring propaganda in the mainstream media (in the age of globalisation and information technologies...). This is obviously part of the antismoking activists' strategy. However, scientific integrity commands that independent researchers avoid such unethical methods. For instance, the credibility of WHO ««waterpipe»»» experts, supervised by the TobReg group, having already published a flawed report on this issue [24], is once again stained when, in the wake of a first world "«waterpipe»» antismoking conference (Oct. 2013), a main leading "«waterpipe»») antismoking expert, and main co-author of the WHO report, dares state in the popular press that shisha is more addictive than a hard drug like cocaine $[24,50]$.

c The "nicotine "addiction"” dogma has been directly and uncritically "translated"/imported from the field of cigarette studies in spite of its flawed premises as knowledgeable scholars highly concerned with scientific integrity have been repeating for almost three decades now [51].

d Since one cigarette puff is supposed to be enough to trigger life addiction [52], ««waterpipe»» antismoking researchers have uncritically carried on several experiments and "meta-analyses" in an endeavour to (try to) establish that shisha produces "substantial" levels of nicotine (even if the corresponding figures are consistently much lower than in cigarettes). While they admit that the use of hookah is often recreational, they stress that young people could nonetheless get addicted and that it would actually represent a "gateway" to cigarette use. There is absolutely no evidence for such gratuitous speculations and exaggerations. Yet, existing data from the field and produced by antismoking researchers themselves, show the opposite [53]. "«Waterpipe»» antismoking researchers, who wish to demonstrate the relevance of the "gateway" theory [53], should not, as they are doing with Ecigarettes, confuse correlation and causation and are invited to carry out longitudinal surveillance [54]. Yet, recent search in the USA relevantly found that: "hookah was the most tried product (38\%), but cigarettes were most often the first product ever tried (51\%). First product tried did not predict current tobacco use and non-use, but individuals who first tried SLT [smokeless tobacco] or cigarettes (rather than hookah or ETPs [Emerging Tobacco Products]) were more likely to be poly tobacco users [...] However, uptake of ETPs is poor, unlike cigarettes and SLT, and does not appear to lead to significant daily/non-daily use of cigarettes and SLT." [55].

e Studies on nicotine and its metabolites have been, in most (if not all) cases, carried out on volunteers without distinguishing between smokers of non addictive smoking mixtures (such as, e.g., flavoured moassel/tabamel) and those of other products (such as tumbak and jurak for example) (See Figs. 1, 2, 3) and even without taking into account the fact, of utmost importance, that some of the volunteers were ex-cigarette smokers having switched to shisha smoking [56]. Therefore, most (if not all) studies (and the corresponding "meta-analyses" and "systematic reviews") so far are stained with a serious methodological flaw $[37,57]$. Referring to a so-called ««waterpipe»» dependence scale" is highly misleading and adding fuel to the global confusion. Independent researchers should meditate on how Karl Fagerström himself, the very scientist whose name was given to the famous test (Fagerström Test for Nicotine Dependence) and on which the above mentioned scale is based, finally admitted that "nicotine "addiction"" is not equivalent to tobacco dependence [58]. 


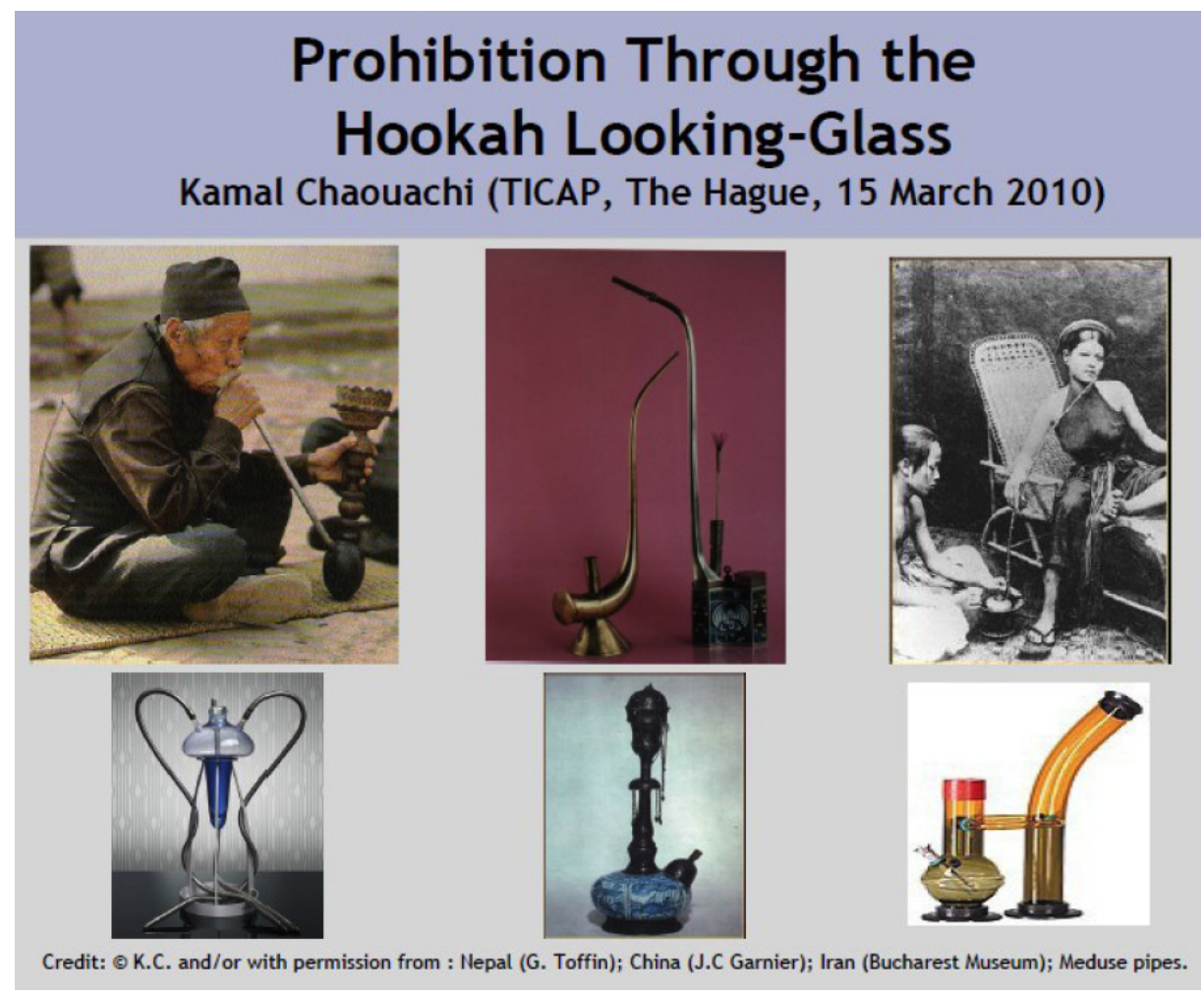

Fig. (5). Different devices produce, in each case, a qualitatively different chemical smoke and induce different health effects, particularly in case of misuse as this happens with inexperienced users. Even if some health effects may be similar to those affecting cigarette smokers, this is generally due to a specific chemical or group of chemicals. This does not mean that all water filtered tobacco smoking pipes do cause the "same effects" as cigarettes.

In sum, ««waterpipe»» nicotine "addiction" sounds as one of the greatest fallacies in tobacco research... On one hand, the research community and the broad public alike have to admit that we would be in presence of a 400 year-old "global "epidemic". On the other, the authors of the WHO flawed report on ««waterpipe»») still are at variance about whether its users are dependent or not... For some, WFTSP would be "addictive" because "some [sic, actually less than $5 \%$ in the world...] smokers experience withdrawal" [59]. For others, there would be "many users" (sic) who "exhibit signs of dependence") [60].

\section{THE EPIDEMIOLOGIC CONCEPT OF PREVA- LENCE HAS BEEN REVISITED}

Just as the definition of an "epidemic" has been misrepresented, there is now a prevalent abuse of another key notion: that of prevalence. Unlike cigarettes, oriental pipes are rather used in a sporadic way. Even under its popularised modern flavoured-moassel/tabamel based form outside Asia and Africa (See Fig. 2), it is used one to three times a week on average. Consequently, it is clear that its users cannot be "exposed to smoke over a longer period of time than a typical cigarette" [61], not to mention the different smoke chemistry as stressed before and backed with several examples. More and more frequently, and as a result of over-citing biased or false results [62] particularly a ten-year old "review" full of scientific errors, which paved the way to the WHO flawed report [24, 63, 64], prevalence figures are sometimes compared in a deceiving way. For instance, one of the chief authors of the above mentioned report stated somewhere that in Jordan the prevalence "was more than double that of cigarette". Now, far from such a country, most researchers will not necessarily understand that shisha is not smoked as frequently as a cigarettes but, on average, dozens of times less.

The dissimulation of such elementary facts keeps in tune with other in the field of toxicity. For instance, the total phenolic compounds found by the German antismoking team were $205 \mu \mathrm{g}$ ( $v s .73 \mu \mathrm{g}$ for a single cigarette) [8]. Admitting the figures actually reflected unbiased measures, this would mean that a single WFTSP session is "equivalent" (considering the toxicity of phenols only) to only 3 cigarettes. If we now take into consideration the actual smoking frequency, considering a usual smoker of 20 cigarettes a day (and that of a shisha smoker of between 0.14 pipe per day and 0.43 pipe a day), the purported toxicity of WFTSP appears to be of a completely different order of magnitude... In this context, it is amazing to read from the German antismoking team itself that phenolic compounds (as measured by themselves [8]) are cause for concern" even more that some of them are completely dissolved in the water tank.

\section{ETHICAL ISSUES}

\subsection{The Coffee, Tea and Barbecue "Global Epidemics"}

In the light of the above discussed issues, the first ethical concern may take the form of a simple question. May one picture what would be the reaction of the scientific community if, tomorrow, scientists succeeded in publishing in the biomedical peer-reviewed literature claims that coffee and tea, or even the barbecue, have actually become "global 

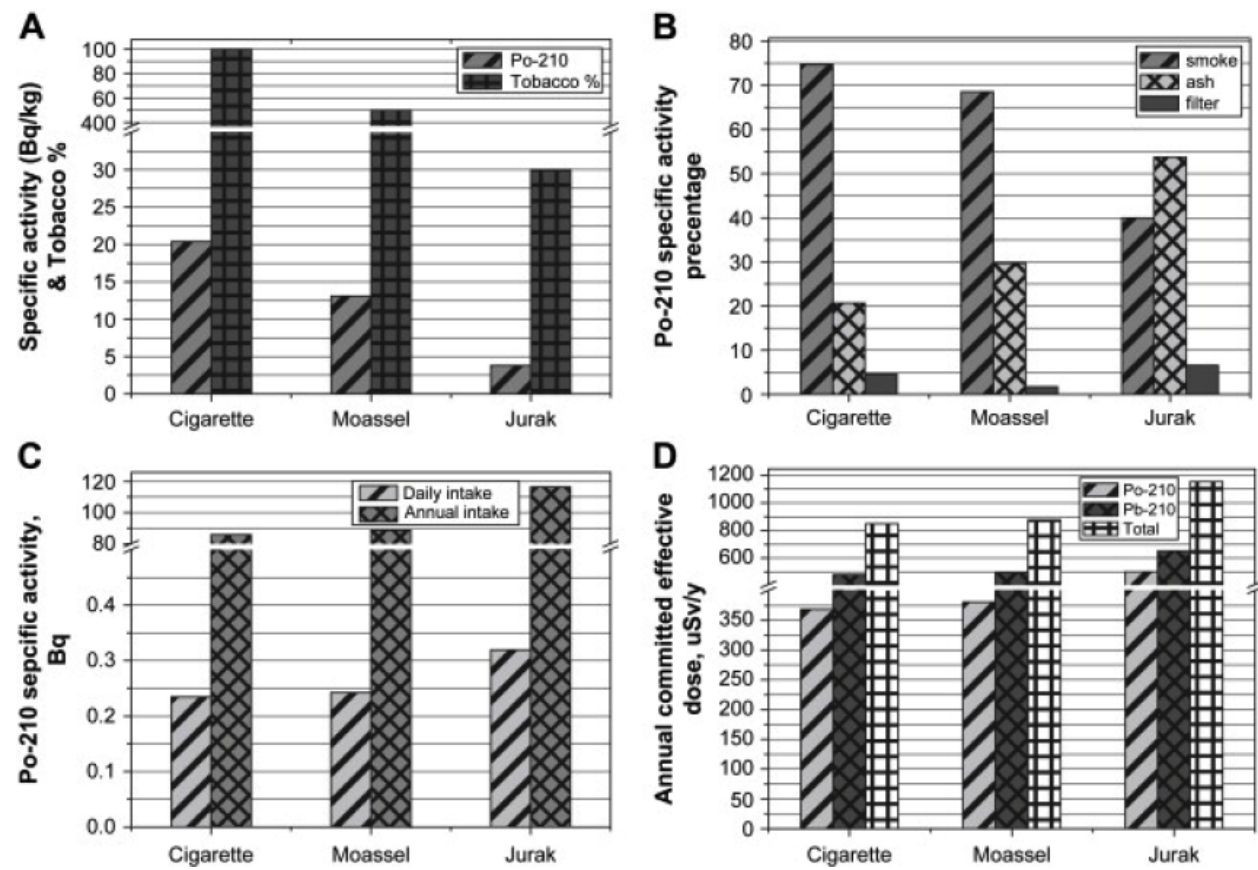

Fig. (6). Activity concentration of 210Po and tobacco content in cigarette, moassel and jurak (a), 210Po activity percentage released in smoke and remained in ash and filter (b), daily and annual intake of Po-210 activity, Bq, due to cigarette, moassel and jurak smoking (c) and annual committed effective dose due to 210Po and 210Pb intake via smoking (source: Khater AE, Abd El-Aziz NS, Al-Sewaidan HA, Chaouachi K. Radiological hazards of Narghile (hookah, shisha, goza) smoking: activity concentrations and dose assessment. J Environ Radioact. 2008 Dec; 99(12): 1808-14).

epidemics"? The question is even more relevant that coffee and tea are known to have spread across the world by the same time in history as WFTSP $\left(16^{\text {th }}-17^{\text {th }}\right.$ centuries $)[1]$, and that the barbecue itself produces smoke and is known for generating great quantities of toxic chemicals like PAHs, $\mathrm{CO}$ and benzene $[3,4]$. In view of the absence of reaction on behalf of researchers, ethics committees outside or inside scientific editorial groups, one can easily conclude that, as far as oriental pipes are concerned, the issue seems affected by double standards.

\subsection{Measuring Poor Hygiene Instead of Actual Health Effects}

It appears that biomedical research ethics is seriously breached when, and just to take one example, toxicity studies hype the risk of cancer (including in the mainstream media) supposedly associated with WFTSP use. The problem is that the corresponding statements are "supported" by experiments in which the water of the "«waterpipe»») was not changed between sessions [5, 27]. Even the question of the violated protection of human subjects was once raised in relation to an amazing experiment in which, not only water had not been changed but also, ventilation not secured [28, 29]. The bottom line is that, most of the time, what such studies actually measure is certainly not the toxicity of hookah smoking "per se" but the risk of poor hygiene, or misuse, including "modelled" misuse as the US-AUB smoking robot based experiments show $[1,5]$.

\subsection{Bibliometric Performance}

Then there is the question of over-citing biased erroneous publications in the available abundant ««waterpipe»» litera- ture. For instance, when the authors of a Jordanian study (coming up with negative results) cite, in a European public health journal, the publications of the US-SCTS up to 80 times in a manuscript of only 6 pages, and that 64 of such citations concern one chief author, namely the head of that organisation, and that it appears up 22 times in the first two paragraphs, this actually raises ethical concerns $[14,64]$. The point here is that such breaches are not isolated cases but have been representing the actual trend for one decade now. This topic takes a renewed importance because some parties are now trying to draw a "bibliometric" picture of research in this field [65]. Yet, what is actually measured in such a project is an artefact due to a methodological vicious circle. Such an unnoticed artefact allows an unscientific article to be cited up to 200 times, not to mention the monopoly of research as the imposed model of the US-AUB smoking robot shows [1].

\subsection{Three Other Phenomena}

Three phenomena are a direct result of over-citing "cherry-picked" research in this field. The first one is the open dismissal, on no scientific ground, of peer-reviewed scientific publications (bibliographical bias). Most of the time, the obvious reason is that the corresponding literature presents negative results even if the related studies represent substantial documents such as, medicine theses, particularly those focussing on lung function, not to mention an early comprehensive transdisciplinary doctoral thesis [1, 66, 67]. Such a bias is what a world renowned epidemiologist, who issued a famous "plea for epistemological modesty", described as the "tendency of authors and journal reviewers and editors to report and publish "positive" or "statistically significant" results over "null" or "non-statistically sig- 
nificant" results, particularly if the findings appear to confirm a previously reported association (i.e., the "bandwagon effect")". As with other forms of bias, preferential publication generates a false sense of consistency among studies" [68]. However, one example of such "false-positive" results was recently highlighted in the biomedical press and involved the author of the above critical statement himself [5]. Unsurprisingly, the research community should be aware that such ethical breaches have also led to a second problem represented by actual cases of plagiarism in "«waterpipe»»" research, be the latter based on direct copying or paraphrasing as the Elsevier Group notes [69]. Ideally, and in agreement with recommendations of ethics committees, cases of plagiarism are generally solved in a "diplomatic" way: e.g., through a Letter to the Editor forcing the faulty authors to openly cite the work they first decided to ignore. This has been done in relation to the core reference cited in the present article $[1,70,71]$. Then, we have a third ethical consequence, unprecedented in the biomedical field. It relates to the history of science since it was arbitrarily announced that research on WFTSPs has actually begun by year 2002 [37]. Interestingly, the last date matches the beginning of activity at the US-AUB and US-SCTS. Of course, this is untrue since research in this field has been documented back to 1622 [72]. The obvious objective of such a rewriting of research history in this field was to make tabula rasa of a long tradition of independent (from both the tobacco and pharmaceutical industries) valuable medical research of the past decades. During such a golden period, pioneers of tobacco science such as Dietrich Hoffmann, Ernst Wynder, Angel Roffo and many other physicians and cancer specialists, particularly in Asia and Africa, did study -objectively and independentlyhookah, narghile and shisha smoking. The dismissal of such figures was striking in the WHO flawed report $[11,24]$. In a normal situation and even assuming (absurd hypothesis) that the world were facing a "global "epidemic"", the first logical step should have been to clarify to what extent the "new" findings, i.e. since 2002, are in agreement, or not, with those published by the above mentioned great names of tobacco research in this field. Amazingly, this has never been done. The clear reason, which has nothing to do with science, is that, most of the time, the pioneers of investigation in this field came up with negative results in their studies (from smoke toxicity to cancer and lung function). The most recent evidence for this regrettable trend is that even when "«waterpipe»»» antismoking teams themselves get negative results, they are not cited in the available literature [19].

\subsection{Epistemological Contradiction of ««Waterpipe»» Research}

Any independent observer is entitled to ask why ««waterpipe»») antismoking teams, apparently so concerned with the health of populations, have been publishing alarming papers on only one type of WFTSP and definitely ignored all the others particularly adapted for tumbak, jurak, etc. (See Figs. 1, 3, 5). Yet, the smokers of the latter are actually much more numerous than those of the former type. The contradiction is striking since a neologism as ««waterpipe», which has led astray so many physicians and epidemiologists in the world, is supposed to cover all WFTSPs of the world: from hookah to narghile, shisha, etc., and all of the corresponding products consumed in them. What is even more concerning is that not only the latter's use (i.e. traditional severalcenturies old) is much more important (prevalent) but they are most of the time served in a mixed way in the very places where studies have been carried out (e.g., coffee houses...). A rare independent study from Kuwait exemplifies this point [15]. It is once again amazing that the latter fact (qualifying for another serious methodological flaw) is silenced in most of the corresponding publications. Indeed, the chemistry of smoke and the potential health effects are completely different in both cases (e.g., between flavoured and unflavoured shisha smoking, not only because the products are different but also because of the set up involving or not a direct contact between the heating or burning source and the smoking product (See Figs. 1, 2, 3).

Finally, in a fair number of cases, researchers draw totally irrelevant parallels with other studies (which are about a completely different product) as this happened recently in such a country as Iran $[39,40]$. One solution to such a great multi-dimension methodological flaw would be that, in each given situation, the researchers specify what kind of water pipe (in two words) is referred to. It just varies, from one setting to other and from one country to other and from one group of users to other. As a consequence, it is clear that it is not a mere matter of "vocabulary" preference which would be easily fixed by adding one to three words "(narghile, hookah, shisha)" in the title of an article in order to make it suddenly and scientifically sound. The clarification must be made from the outset, in the abstract itself and in the introduction, methods, discussion, results sections and conclusion of each publication. For instance, the authors should state something like: "In this report, we have studied smokers of this type of pipe and product (e.g., moassel/tabamel) (See Fig. 2). We have or not distinguished them from the users of this or that other product (e.g. tumbak, jurak) (See Figs. 1, 3). Moreover, a proportion of $\mathrm{x} \%$ of our smokers were previous cigarette users and $\mathrm{y} \%$ were actually smokers who had switched -in a more or less recent past- from cigarette to this or that type of tobacco or tobacco-based product. There are also other details of utmost importance such as the use -or not- of a thermal screen and of what type (aluminium, zinc plate, vaporising bowl, etc.) and, of course, the classical and not less key questions about hygiene: e.g., "how often do you change the water" [5]. Any infringement to such basic methodological rules amounts to a form of reductionism (of a complex issue) and, therefore, needs to be exposed.

\section{CONCLUSION}

Thanks to striking examples of serious contradictions exposed here and in a previous article [5], we have shown that most toxicity studies have actually demonstrated "how NOT to use" WFTSPs... Scientific ethics commands that public health organisations should stop delaying the dissemination of practical minimal recommendations to millions of WFTSP users across the world. Just calling for "eradication" (sic) or immediate "implementation" of a prohibitionist agenda of the FCTC type [9, 10, 22, 25, 35], is reminiscent of those self-righteous activists who, one century ago in the USA, pushed for alcohol prohibition. In the end, it proved to be a total human, economic and socio-cultural failure. Research on WFTSPs should recover the independence that it 
has unfortunately lost one decade ago and remain open to all views, particularly those independent from the African and Asian continents, and not only to a heavily funded elite who has not brought so far the least evidence of its alleged excellence. Yet, the opposite is true. The first step should be to address the numerous ethical breaches reviewed before. The publication of negative results should be encouraged alongside positive findings so that the practice of public health be enhanced for the benefit of populations before that of researchers [1]. For example, a recent spontaneous Letter to the Editor by researchers in Thailand is a model to follow [73]. By their independent discussion about the importance of dose-response aspects and genetic factors, the authors are reminiscent of others who openly, and as early as 1955, discussed in the Lancet, the possibility of a null, low or reduced risk of cancer among narghile smokers [12]; a hypothesis positively tested seven years and half a century later, respectively $[11,13]$. A key message to be disseminated to both users and non-users of WFTSPs is that the great majority of the toxicity ( $90 \%$ and much more) comes from the charcoal in a way very similar to the use and misuse of barbecues. On one hand, important amounts of tobacco may be consumed in WFTSPs ("up to $375 \mathrm{~g}$ tobacco") [43]. On the other, the quality of this (coarse) tobacco is generally low, particularly in Asia and Africa. A reasonable anthropological conclusion can now be drawn from these two facts and the toxicological and medical considerations reviewed in this article [5, 11]. WFTSPs (from hookahs to narghiles and from shishas to mada'i) would represent the oldest tobacco harm reduction technique in the world. It has been a natural one, long before the arrival of the E-cigarette which, even it may be more efficient, is based on the principle of the former: vapourising nicotine and flavours $[5,11,20]$.

\section{ABBREVIATIONS}

$$
\begin{aligned}
\text { WFTSPs }= & \text { Water Filtered Tobacco Smoking Pipes } \\
\text { US-AUB = } & \text { US American University of Beirut } \\
\text { US-SCTS }= & \text { US-Syrian Centre for Tobacco Studies in } \\
& \text { Aleppo }
\end{aligned}
$$

\section{CONFLICT OF INTEREST}

The author declares that he has no competing interests. He has never received financial or non-financial, direct or indirect, funding either from pharmaceutical companies (nicotine "replacement" therapies and products) or from the tobacco industry. He has been, at times between years 2000 and 2007, an active member of the world antismoking Globalink network sponsored by the pharmaceutical industry (Pfizer in particular). This organisation counts some 6,000 members working with: ministries of health; antismoking NGOs; the World Health Organisation and its relevant bodies (TobReg, the Study Group for the Regulation of Tobacco Products; the "Tobacco Free Initiative"; the regional bureaux; etc.); the Cochrane Review Tobacco Addiction Group; etc. Globalink also maintains strong links with the main antismoking journals: "Tobacco Control" most importantly; "Nicotine and Tobacco Research"; "Addiction"; some US biomedical journals which regularly publish articles on tobacco issues, etc. Since some views expressed in the present article could perhaps be seen as influenced by such an experience, the author wishes to confirm that both the scientific presentation of facts and the necessary discussion exclusively rely on the available world peer-reviewed scientific literature." Dr. Kamal Chaouachi is also co-founder of the French non-profit association for independent multidisciplinary research (medicine, toxicology, history, psychology, sociology, anthropology, economics, etc.) on oriental pipes.

\section{ACKNOWLEDGEMENTS}

Declared none.

\section{PATIENT'S CONSENT}

Declared none.

\section{REFERENCES}

[1] Chaouachi, K. Tout savoir sur le narguilé. Société, culture, histoire et santé [Eng.: Everything about hookahs. society, culture, origins and health aspects], $2^{\text {nd }}$ ed.; L'Harmattan: Paris, 2012. Previously published by Maisonneuve \& Larose, Paris, 2007, ISBN: 978-27068-1954-4. Preface by Pr Robert Molimard. Extract from the first transdisciplinary doctoral thesis on this issue.

[2] Yüksel, Hülya. Tütün kontrolünde neolýberal polýtýkalarin yansimasi ve saðlik eðýtýmý [The influence of neoliberal policies on "tobacco "control"” and health education]. Dumlupýnar Üni. Sosyoloji Bölümü. 2014, 11 pages, [Accessed 8 Dec. 2014]. https://www.academia.edu/4007251/The_Influence_of_Neoliberal_ Policies_on_Tobacco_Control_and_Health_Education

[3] Olsson, M.; Petersson, G. Benzene emitted from glowing charcoal. Sci. Total Environ., 2003, 303, 215-220.

[4] Rahman, M. M.; Kim, K. H. Release of offensive odorants from the combustion of barbecue charcoals. J. Hazard Mater. 2012, 215-6, 233-242.

[5] Chaouachi, K. False positive result in study on hookah smoking and cancer in Kashmir: measuring risk of poor hygiene is not the same as measuring risk of inhaling water filtered tobacco smoke all over the world. Br. J. Cancer., 2013, 108, 1389-1390.

[6] Chaouachi K. Public health intervention for narghile (hookah, shisha) use requires a radical critique of the related "standardised" smoking machine. J. Public Health, 2009, 18, 69-73.

[7] Chaouachi, K. Assessment of narghile (shisha, hookah) smokers' actual exposure to toxic chemicals requires further sound studies. Libyan J.of Med., 2011, 6, 5934-5939.

[8] Schubert, J.; Müller, F.D.; Schmidt, R.; Luch, A.; Schulz, T.G. Waterpipe smoke: source of toxic and carcinogenic VOCs, phenols and heavy metals? Arch. Toxicol., 2014, Sep 24. [Epub ahead of print]

[9] Nakkash, R.; Afifi, R.; Maziak, W. Research and activism for tobacco control in the Arab world. Lancet, 2014, 383, 392-393.

[10] Maziak, W.; Nakkash, R.; Bahelah, R.; Husseini, A.; Fanous, N.; Eissenberg, T. Tobacco in the Arab world: old and new epidemics amidst policy paralysis. Health Policy Plan., 2013, Doi:10.1093/heapol/czt055

[11] Sajid, K.M.; Chaouachi, K.; Mahmood, R. Hookah smoking and cancer. Carcinoembryonic antigen (CEA) levels in exclusive/ever hookah smokers. Harm. Reduct. J., 2008, 5-19. http://www.pubmedcentral.nih.gov/articlerender.fcgi?tool=pubmed \&pubmedid=19440416

[12] [Anon.] Annotations, The Lancet, 1955, 392-3.

[13] Rakower, J.; Fatal, B. Study of Narghile Smoking in Relation to Cancer of the Lung. Br. J. Cancer, 1962, 16, 1-6.

[14] Al-Safi, S.A.; Ayoub, N.M.; Albalas, M.A.; Al-Doghim, I.; AboulEnein, F.,H. Does shisha smoking affect blood pressure and heart rate ?. Public Health, 2009, 17, 121-126.

[15] Al Mutairi, S.S.; Mojiminiyi, O.A.; Shihab-Eldeen, A.A.; Al Sharafi, A.; Abdella, N. Effect of smoking habit on circulating adipokines in diabetic and non-diabetic subjects. Ann. Nutr. Metab., 2008, 52, 329-334.

[16] Khater, E.M.; Amr, M.; Chaouachi, K. Elemental characterization of shisha moassel smoking mixtures using ICP-MS and comparison with other tobacco products. Wulfenia J., 2014, 21, 428-449. 
[17] Ben Saad, H.B.; Khemiss, M.; Nhari, S.; Essghaier, M.B.; Rouatbi, S. Pulmonary functions of narghile smokers compared to cigarette smokers: a case-control study. Libyan J. Med., 2013, $8,1-8$.

[18] Mohammad, Y.; Shaaban, R.; Abou Al-Zahab, B.; Khaltaev, N.; Bousquet, J.; Dubaybo, B. Impact of active and passive smoking as risk factors for asthma and COPD in women presenting to primary care in Syria: first report by the WHO-GARD survey group. Int. J. Chron. Obstruct. Pulmon.. Dis., 2013, 8, 473-482.

[19] Apsley, A.; Galea, K.S.; Sánchez-Jiménez, A.; Semple, S.; Wareing, H.; Van Tongeren, M. Assessment of polycyclic aromatic hydrocarbons, carbon monoxide, nicotine, metal contents and particle size distribution of mainstream Shisha smoke. J. Environ. Health Res., 2011, 11, 93-103.

[20] Chaouachi, K. Hookah (shisha, narghile) smoking and environmental tobacco smoke (ETS). A critical review of the relevant literature and the public health consequences. Int. J. Environ. Res. Public Health, 2009, 6, 798-843. http://www.pubmedcentral.nih.gov/ articlerender.fcgi?tool=pubmed\&pubmedid $=19440416$

[21] Chaouachi, K. Is medical concern about hookah environmental tobacco smoke hazards warranted ? [A Tribute to Gian Turci, who has recently passed away]. The Open Gen. Internal Med. J., 2009, 3, 31-3.

[22] Bahelah, R. Waterpipe tobacco labeling and packaging and World Health Organization Framework Convention on Tobacco Control (WHO FCTC): a call for action. Addiction, 2013, S0091-3057.

[23] ASH (Action on Smoking and Health). "“Shisha 200 times worse than a cigarette" say Middle East experts"”. 2007 (prepared by Martin Dockrell)[accessed 13 June, 2008]. Based on an interview with Wasim Maziak and Alan Shihadeh. Sub-heading: "Three leading experts from across the Middle East have warned that excluding "shisha bars" when England goes smokefree on July 1 could worsen the grave inequalities in health that already affect ethnic minorities". [Accessed 8 Dec. 2014]. http://www.ash.org.uk/ mediaroom/press-releases/shisha-200-times-worse-than-a-cigarette- saymiddle-east-experts

[24] Chaouachi, K. A Critique of the WHO's TobReg "Advisory Note" entitled: "Waterpipe Tobacco Smoking: Health Effects, Research Needs and Recommended Actions by Regulators. J. Negat. Results Biomed., 2006, 5, 1-17. http://www.ncbi.nlm.nih.gov/pmc/ articles/PMC1664583/

[25] Jawad, M.; Bakir, A.M.; Ali, M.; Jawad, J.; Akl, E.A. Key health themes and reporting of numerical cigarette-waterpipe equivalence in online news articles reporting on waterpipe tobacco smoking: a content analysis. Tob. Control, 2015, 24, 43-47.

[26] Djordjevic, M.V.; Doran, K.A. Nicotine content and delivery across tobacco products. Handb. Exp. Pharmacol., 2009, 192, 6182.

[27] Jacob, P.; Abu Raddaha, A. H.; Dempsey, D.; Havel, C.; Peng, M.; $\mathrm{Yu}, \mathrm{L}$.; Benowitz, N.L. Comparison of nicotine and carcinogen exposure with water pipe and cigarette smoking. Cancer Epidemiol. Biomarkers Prev., 2013, 22, 765-772.

[28] Chaouachi, K. Hookah (shisha, narghile, "water pipe") indoor air contamination in German unrealistic experiment. Serious methodological biases and ethical concern. Food Chem. Toxicol., 2010, 48, 992-995.

[29] Fromme, H.; Dietrich, S.; Heitmann, D.; Dressel, H.; Diemer, J.; Schulz, T.; Jörres, R.A.; Berlin, K.; Völkel, W. Indoor air contamination during a waterpipe (narghile) smoking session. Food Chem Toxicol., 2009, 47, 1636-1641.

[30] Nassar, A.H.; Abu-Musa, A.; Hannoun; A.; Usta, I.M. Authors' reponse: nargile smoking and its effect on in vitro fertilization: a critical eye on the available literature. Eur. J. Obstet. Gynecol., 2010, $152,116$.

[31] Underwood M. Shisha: The Middle East's favourite toxin. The National (Emirates), 2013. [Accessed 8 Dec. 2014]. http://www.thenational.ae/uae/health/shisha-the-middle-eastsfavourite-toxin\#full

[32] CDC. Evidence: New CDC study finds dramatic increase in ecigarette-related calls to poison centers. [Accessed 8 Dec. 2014]. http://www.cdc.gov/media/releases/2014/p0403-e-cigarettepoison.html

[33] Goldman, A.L. Carboxyhemoglobin levels in primary and secondary cigar and pipe smokers. Chest, 1977, 72, 33-35.

[34] Rylander, R. Environmental Tobacco Smoke Effects on the Nonsmoker. Workshop; Bermuda, March 27-29, University of Geneva: Geneva, Switzerland, 1974
[35] Kassem, N.O.; Daffa, R.M.;Liles, S.; Jackson, S.R.; Kassem, N.O.; Younis, M.A.; Mehta, S.; Chen, M.; Jacob, P.3rd; Carmella, S.G.; Chatfield, D.A.; Benowitz, N.L.; Matt, G.E.; Hecht, S.S.; Hovell, M.F. Children's Exposure to Secondhand and Thirdhand Smoke Carcinogens and Toxicants in Homes of Hookah Smokers. Nicotine Tob. Res., 03 March 2014. Doi: 10.1093/ntr/ntu016.

[36] Saadawi, R.; Figueroa, J.A.L.; Hanley, T.; Caruso, J. The hookah series part 1: total metal analysis in hookah tobacco (narghile, shisha) —an initial study. Ana.l Methods, 2012, 4, 3604-3611

[37] Chaouachi, K.; Sajid, K.M. A critique of recent hypotheses on oral (and lung) cancer induced by water pipe (hookah, shisha, narghile) tobacco smoking. Med. Hypotheses, 2010, 74, 843-846.

[38] Layoun, N.; Saleh, N.; Barbour, B.; Awada, S.; Rachidi, S.; AlHajje, A.; Bawab, W.; Waked, M.; Salameh, P. Waterpipe effects on pulmonary function and cardiovascular indices: a comparison to cigarette smoking in real life situation. Inhal. Toxicol., 2014, 26, 620-627.

[39] Boskabady, M.H.; Farhang, L.; Mahmodinia, M.; Boskabady, M.; Heydari, G.R. Comparison of pulmonary function and respiratory symptoms in water pipe and cigarette smokers. Respirology, 2012, $17,950-956$.

[40] Chaouachi, K. Lung function of water pipe (Qalyan, Narghile, Shisha, Hookah) users $v s$. cigarette smokers in Iran [Critical analysis of the study]. Tabaccologia, 2013, 3, 18-29.

[41] Schulz, T.; Dettbarn, G.; Völkel, W.; Hahn, J. Water pipe smoking: biomarkers of exposure. Audiovisual presentation at $14^{\text {th }}$ World Congress on Tobacco or Health (WCTOH), Bombay, 2009, March 9. Accessed Jan. 2010. http://www.14wctoh.org/abstract/ abs detail.asp?AbstractID $=434$

[42] Chaouachi, K.; Sajid, K.M. Cancer risks of hookah (shisha, narghile) tobacco use require further independent sound studies. Int. $J$ Cancer, 2010, 27, 1737-1739.

[43] Muzaffar, M., Dar, N.A. Esophageal cancer in Kashmir (India): An enigma for researchers. Int. J. Health Sci. (Qassim), 2009, 3, 71-85.

[44] Khlifi, R. Response to comment of Dr. Kamal Chaouachi on "Shisha Smoking, Nickel and Chromium Levels in Tunisia". Environ. Sci. Pollut. Res. Int., 2013, 20, 82-97.

[45] Billard, G.; Chaouachi, K.; De La Giraudiere, A.-P. Hookah with simplified lighting. US Patent Application, 2005, 2005/0279371 A1. Application number 11/148, 194.

[46] Bertholon, J.F.; Becquemin, M.H.; Roy, M.; Roy, F.; Ledur, D.; Annesi Maesano, I.; Dautzenberg, B. Comparaison de l'aérosol de la cigarette électronique à celui des cigarettes ordinaires et de la chicha [Comparison of the aerosol produced by electronic cigarettes with conventional cigarettes and the shisha]. Rev. Mal. Respir., 2013, 30, 752-757.

[47] Torrey, C.M.; Moon, K.A.; Williams, D.A.; Green, T.; Cohen, J.E.; Navas-Acien, A.; Breysse, P.N. Waterpipe cafes in Baltimore, Maryland: Carbon monoxide, particulate matter, and nicotine exposure. J. Expo. Sci. Environ. Epidemiol. 2014, Apr 16. doi: 10.1038/jes.2014.19. [Epub ahead of print]

[48] Kabat, G. Is "Thirdhand Tobacco Smoke" a valid scientific concept or a public relations gimmick? The Columbia University Press Blog, 2009. [Accessed 08 Dec. 2014] http://www.cupblog.org/? $\mathrm{p}=493$ (accessed 8 Dec. 2014)

[49] Salameh, P.; Aoun, Z.; Waked, M. Saliva cotinine and exhaled carbon monoxide in real life narghile (waterpipe) smokers: a post hoc analysis. Tob. Use Insights, 2009, 2, 1-10.

[50] Al-Taher, N. Shisha smoking is more addictive than cocaine, expert says. Expert says those who smoke hookah likely to switch to cigarettes within three years. Gulf News, 26 Oct. 2013. [Accessed 08 Dec. 2014]. http://m.gulfnews.com/news/uae/general/shisha-smokingis-more-addictive-than-cocaine-expert-says-1.1247504

[51] Molimard, R. The myth of nicotine addiction. Tabaccologia, 2013 3, 18-29.

[52] Dar, R.; Frenk, H. Can one puff really make an adolescent addicted to nicotine? A critical review of the literature. Harm Reduct. J., 2010, 7, 10-31.

[53] Carroll, T.; Poder, N.; Perusco, A. Is concern about waterpipe tobacco smoking warranted? Aust. N. Z. J. Public Health., 2008, 32, 181-182.

[54] Abrams, D.B. Potential and Pitfalls of e-Cigarettes-Reply. JAMA, 2014, 311, 1922-1923.

[55] Meier, E.M.; Tackett, A.P.; Miller, M. B.; Grant, D.M.; Wagener, T. L. Which nicotine products are gateways to regular use?: First- 
Tried tobacco and current use in college students. Am. J. Prev. Med., 2015, 48, 86-93.

[56] Macaron, C.; Macaron, Z.; Maalouf, MT.; Macaron, N.; Moore, A. Urinary cotinine in narguila or chicha tobacco smokers. J. Med. Liban., 1997, 45, 19-20.

[57] Chaouachi, K. More Rigor needed in systematic reviews on "Waterpipe" (hookah, narghile, shisha) smoking. Chest, 2011, 139, 1250-1251.

[58] Molimard, R. Fagerstromo trovas sian vojon al Damasko ["Fagerström finds his way to Damascus" (translated by Iro Cyr))]. 26 Nov 2011. [Accessed 08 Dec. 2014]. http://www.formindep.org/ Fagerstromo-trovas-sian-vojon-al.html

[59] Aboaziza, E.; Eissenberg, T. Waterpipe tobacco smoking: what is the evidence that it supports nicotine/tobacco dependence? Tob. Control, 2014. Doi: 10.1136/tobaccocontrol-2014-051910

[60] Asfar, T.; Al Ali, R.; Rastam, S.; Maziak, W.; Ward, K.D. Behavioral cessation treatment of waterpipe smoking: The first pilot randomized controlled trial. Addict. Behav., 2014, 39: 1066-1074.

[61] Dave, B. Why do GDPs fail to recognise oral cancer? The argument for an oral cancer check list. Br. Dent. J., 2013, 214, 223-225.

[62] West, R.; Mcllwaine, A. What do citation counts count for in the field of addiction? An empirical evaluation of citation counts and their link with peer ratings of quality. Addiction, 2002, 97, 501504.

[63] Maziak, W.; Ward, K. D.; Afifi Soweid, R.A.; Eissenberg, T. Tobacco smoking using a waterpipe: a re-emerging strain in a global epidemic. Tob. Control, 2004, 13, 327-333.

[64] Chaouachi, K. Errors and misquotations in the study of shisha, blood pressure and heart rate in Jordan. J. Pub. Health, 2009, 17, $355-356$.
[65] Zyoud, S.H.; Al-Jabi, S.W.; Sweileh, W.M.; Awang, R. A scopusbased examination of tobacco use publications in Middle Eastern Arab countries during the period 2003-2012. Harm Red. J., 2014, 11, 1-14.

[66] Khalsi, S. Les manifestations respiratoires liées à la consommation chronique de narguilé : à propos de 30 cas [Respiratory manifestations of narghile chronic use]. Thèse de médecine, Tunis, 2005, 56.

[67] Mejri, A. Le Tabagisme par le narguilé [Narghile and tobacco abuse]. Thèse de médecine, Tunis, Faculté de médecine, 1985, 41.

[68] Boffetta, P.; McLaughlin, J. K.; La, V.C.; Tarone, R.E.; Lipworth, L.; Blot, W. J. False-positive results in cancer epidemiology: a plea for epistemological modesty. J. Cancer. Inst., 2008, 100: 988-995.

[69] Elsevier Group Plagiarism. Ethics in Research \& Publication. Accessed 08 Dec. 2014 www.ethics.elsevier.com/pdf/ETHICS_PLA01a.pdf

[70] Chaouachi, K. An open letter against plagiarism and plagiarists. Tabaccologia, 2009, 1, 46-47.

[71] Zaga V. Plagiarism in biomedical sciences: a bad habit that needs to be rooted out [Il plagio in campo medico-scientifico: un malcostumbre da estirpare]. Tabaccologia, 2009, 4, 5-7.

[72] Chaouachi, K. Did pre-Columbian mummies smoke tobacco? Evidence in the light of most recent tobaccological \& anthropological findings [Una revisione critica degli elementi di prova ala luce delle conclusioni tabaccologiche e antropologiche più recenti]. Tabaccologia, 2012, 1-2, 31-46.

[73] Wiwanitkit, S.; Wiwanitkit, V. Shisha versus cigarette smoking and endothelial function. Anadol. Kardiyol. Derg., 2014, May 6. Doi: 10.5152/akd.2014.5410. [Epub ahead of print].

(C) Kamal Chaouachi; Licensee Bentham Open.

This is an open access article licensed under the terms of the Creative Commons Attribution Non-Commercial License (http://creativecommons.org/licenses/by-nc/3.0/) which permits unrestricted, non-commercial use, distribution and reproduction in any medium, provided the work is properly cited. 